\title{
Ayur-Informatics: Establishing an Ayurvedic Remedy for Bronchial Carcinoma
}

\author{
${ }^{1 *}$ Preenon Bagchi, ${ }^{2}$ Nikita Sinha, ${ }^{3}$ Ajit Kar, and ${ }^{4}$ Mahesh. M
}

\begin{abstract}
Bronchial carcinoma is the world's most common type of cancer associated with the mutations of ras, sre, and myc genes and tumor suppressor proteins p53 and BRCA1 along with the epidermal growth factor receptors (EGFR). 3D structures of these proteins were generated using homology modeling. Active compounds of the medicinal herbs and plants were selected on the basis of their properties. Chemical structures of the active components of these herbs were drawn using chemsketch, combined and converted to*.pdb. The four proteins were successfully docked with the combination of active components.
\end{abstract}

Index Terms-Bronchial carcinoma, mutation, homology modeling, ayurveda, docking, drug designing.

\section{INTRODUCTION}

Ayur-informatics is a science dealing with the application of bioinformatics to the ayurvedic medication. The traditional ayurvedic medication originated during the vedic periods. Susruta samhita and Charaka samhita are the oldest ayurvedic literatures. According to ayurveda, the disease evolves from the body due to external factors. It has a vast literature in Sanskrit covering all aspects of diseases, pharmacy and therapeutics. The Indian subcontinent is a vast repository of medicinal plants that are used in traditional medical treatments. Many westerners have long regarded the Indian systems of medicine as a rich source of knowledge. In India, around 20,000 medicinal plants have been recorded; however, traditional communities are using only 7000-7,500 plants for curing different diseases [1].

Ayur-informatics is an approach to provide a scientific platform to the traditional Indian medications. There has been an increase in demand for the phytopharmaceutical products of ayurveda in Western countries because of the fact that the allopathic drugs have more side effects.

\section{A. Bronchial carcinoma}

All Bronchial carcinoma is declared a deadly disease; though a lot have been done for the prevention and cure of the disease on the other side. Besides the chemotherapy, radiotherapy and the surgical treatments, few more have been suggested such as gene transplantation. But all these are neither cost effective nor a complete cure for the disease [2].

\footnotetext{
Manuscript received April 1, 2011

${ }^{1}$ Research Scholar, Dept. of Bioinformatics,SRM University, Kattankulathur, Tamil Nadu, India.

${ }^{*}$ Corresponding author e-mail: prithish.bagchi@gmail.com

${ }^{2}$ Student, Department of Computer Science, Jamia Millia Islamia University, New Delhi, India.

${ }^{3}$ Chief Chemist, Satsang Herbal Research Laboratory, S R Mandir, Satsang, Deoghar, Jharkhand, India.

${ }^{4}$ CEO,Azyme Bioscience Pvt. Ltd, 9th Block, Jayanagar, Bangalore, Karnataka, India.
}

The development and progression of lung cancers is a multi-step process characterized by the accumulation of numerous genetic alterations. Studies suggest that p53 and $\mathrm{H}$-ras mutations are frequent and apparently independent genetic alterations which play different roles in the pathogenesis, progression and prognosis of non small cell lung cancer. The functions of BRCA1in DNA damage response provide a reasonable molecular explanation for its role as a tumor suppressor. Compromised functions of BRCA1 in DNA repair and cell cycle checkpoint likely contribute in a significant manner to cancer susceptibility. Respiratory epithelial cells require prolonged exposure to cancer-promoting agents and accumulation of multiple genetic mutations before becoming neoplastic (an effect called field carcinogenesis). Over time, mutations in genes that stimulate cell growth (K-ras, MYC) cause abnormalities in growth factor receptor signaling (EGFR), inhibit apoptosis, and contribute to proliferation of abnormal cells. In addition, mutations that inhibit tumor-suppressor genes (p53) can lead to cancer. The HRAS gene provides instructions for making a protein called H-Ras which is a GTPase, involved primarily in regulating cell division. Through a process known as signal transduction, the H-Ras protein relays signals from outside the cell to the cell's nucleus. These signals instruct the cell to grow or divide. The TP53 gene provides instructions for making a protein called tumor protein $\mathrm{p} 53$. This protein acts as a tumor suppressor, which means that it regulates cell division by keeping cells from growing and dividing too fast or in an uncontrolled way [2, 3, 4, 5].

Sage Charaka, the father of ayurveda, had mentioned many rasayans in his book "Charaka samhita" which is the basis of modern ayurveda. In India, traditional medicinal knowledge is very scientific and rich due to it is in practice since more than 5000 years, where many natural products are used as medicines [1].

If the modern drug designing technologies and bioinformatics are applied to the traditional ayurveda then it will prove to be a boon to the Medical Science.

\section{B. Ayurvedic medications of Bronchial carcinoma \\ 1) D-limonene}

D-Limonene is the major component of the oil extracted from citrus rind. When citrus fruits are juiced, the oil is pressed out of the rind. This oil is separated from the juice, and distilled to recover certain flavor and fragrance compounds. The bulk of the oil is left behind and collected. This is food grade d-Limonene [6].

\section{2) Berberine}

Berberine is an isoquinoline alkaloid with a bright yellow color that is easily seen in most of the herb materials that contain any significant amount of this compound. It is 
isolated from root of Burberis vulgaris Berberine arrests cancer cell cycle in G1 phase and induces apoptosis. It exhibits immunoenhancing, antioxidant and antiinflammatory properties [7].

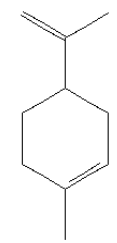

Fig 1. Structure of D-limonene

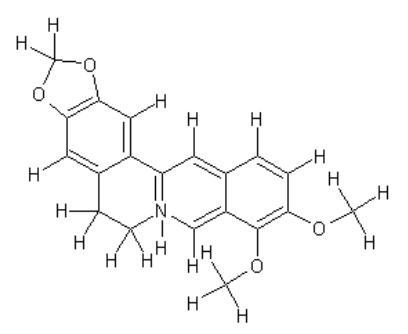

Fig 2. Structure of berberine

\section{3) Curcumin}

Like many herbal remedies, people first used Curcumin as a food and later discovered that it also had impressive medicinal qualities. Over the centuries, this spice has been used as a pain relieving, anti-inflammatory agent to relieve pain and inflammation in the skin and muscles. In modern times, research has focused on Curcumin's antioxidant, antiinflammatory, anticarcinogenic, and antimicrobial properties, and on its use in cardiovascular disease, gastrointestinal disorders, and as a treatment for the liver. In a study conducted by the United States National Cancer Institute, the researchers noted, "Of particular interest for cancer prevention is the role of turmeric (Curcumin), an ingredient in common Indian curry spice. Curcumin is present in Curcumin longa (turmeric). Curcumin arrests the cancer cells proliferation in G2/S phase and induces apoptosis [8].<smiles>[2H]C([2H])([2H])Oc1c(O)cccc1/C=C/C(=O)C/C=C/c1ccc(O)c(O)c1C=O</smiles>

Fig 3. Structure of curcumin

\section{4) Daidzein}

Daidzein is an isoflavone, which is a hormone-like substance found in soybeans. Daidzein is the second most plentiful isoflavone in soy, after genistein. Daidzein inhibits the growth of cancer by taking the place of estrogen on receptors in cancerous cells that need estrogen to grow. Daidzein also inhibits the growth of tumors by forcing cells to differentiate normally, rather than the wild, exponential cell growth seen in tumor cells [9].

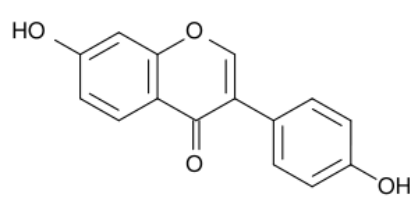

Fig 4- Structure of daidzein

\section{5) Podophyllotoxin}

A lignan (LIGNANS) found in PODOPHYLLIN resin from the roots of PODOPHYLLUM plants. It is a potent spindle poison, toxic if taken internally, and has been used as a cathartic. It is very irritating to skin and mucous membranes, has keratolytic actions, has been used to treat warts and keratoses, and may have antineoplastic properties, as do some of its congeners and derivatives. A pure, stabilized form of podophyllin, in which only the biologically active portion of the compound is present. Podophyllotoxin is a toxic, polycyclic antimitotic agent isolated primarily from the rhizome of the plant Podophyllum peltatum. This agent is formulated for topical applications [10].

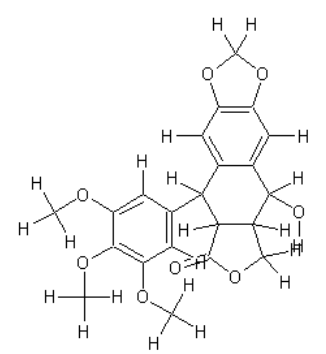

Fig 5. Structure of podophyllin

6) Sulforaphane is a phytochemical belonging to the family of isothiocyanates, which means it contains the typical NCS group. Sulforaphane is an antioxidant and stimulators of natural detoxifying enzymes. Sulforaphane can reduce the risk of breast cancer and prostate cancer [11].

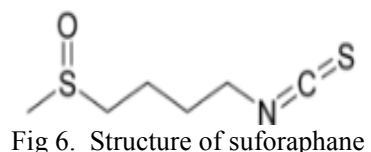

7) Vindoline

Sources: vinca rosea (periwinle madagaskar)

properties It is a vinca alkaloid which acts as a mitotic inhibitor [12]

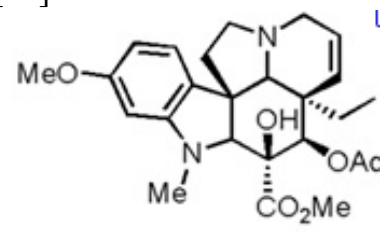

Fig 7. Structure of vindoline

8) Emodin is a biologically active, naturally occurring anthraquinone derivative (1,3,8-trihydroxy-6methylanthraquinone) [13]. 
<smiles>Cc1cc(O)c2c(c1)C(=O)c1cc(O)cc(O)c1C2=O</smiles>

Fig 8. Structure of emodin

9) Ursolic acid is a pentacyclic triterpenoid. It is used in many cosmetic preparations for its anti-inflammatory, antitumor and antimicrobial properties.

Topical application of ursolic acid inhibited TPA-induced initiation and promotion of tumor growth [14].

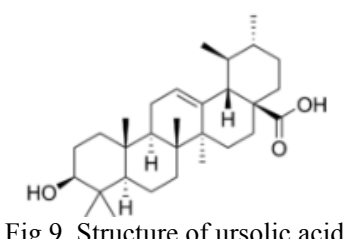

10) Oleanolic acid is a triterpenoid compound that exists widely in food, medicinal herbs and other plants. Oleanolic acid has also been long-recognized to have antiinflammatory and antihyperlipidemic properties in laboratory animals, and more research is warranted to develop a therapy for patients. Recently, it has been noted for its antitumor-promotion effects, which is stimulating additional research in this field [15].

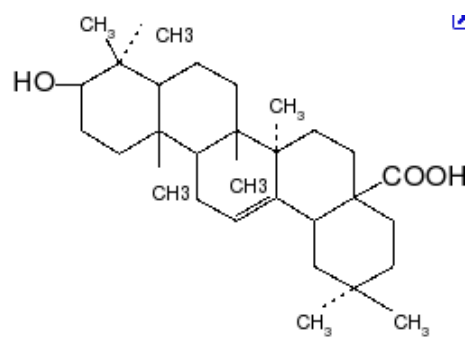

Fig 10. Structure of oleanolic acid

11) Genistein is one of several known isoflavones. Genistein was first isolated in 1899 from the dyer's broom, Genista tinctoria; hence, the chemical name derived from the generic name [16].

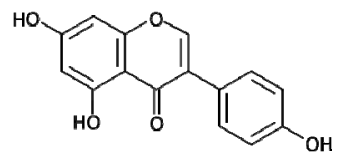

Fig 11. Structure of genistein

12) Epicatechin gallate is a flavan-3-ol, a type of flavonoid, present in green tea. It is also reported in buckwheat and in grape [17].

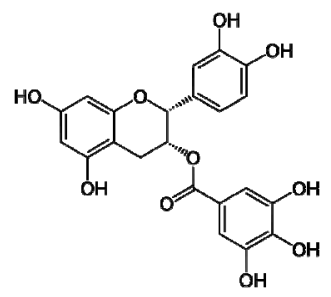

Fig 12. Structure of epicatechin gallate

13) Galangin is a flavonol found in high concentrations in Alpinia officinarum (lesser galangal). It is also found in the galangal rhizome (Alpinia galanga) and in propolis. Galangin has been shown to slow the increase and growth of breast tumor cells [18].<smiles>O=c1c(O)c(-c2ccccc2)oc2cc(O)cc(O)c12</smiles>

Fig 13. Structure of galangin

\section{Methodology}

Homology Modelling was done for the proteins RAS, MYC, SRC, BRCA1, P53 and EGFR using Modeller9v7. For the amino acid sequence of each protein was retrieved from NCBI's database and advance modeling was used (taking three templates for each protein) for modeling the structures. The generated models were verified using Ramachandran plot and best model for each protein was selected. This best model is docked with suitable ligand.

RAS: NCBI Reference Sequence: NP_036351.3

Templates

$$
\begin{aligned}
& \text { 1APA identity }=55 \% \\
& \text { 1C1YA identity }=54 \% \\
& \text { 4Q21A identity }=50 \%
\end{aligned}
$$

SRC: NCBI Reference Sequence: AAH11566.1

1QCFA identity $=61 \%$

$1 \mathrm{AD} 5 \mathrm{~A}$ identity $=60 \%$

1OPKA identity $=43 \%$

MYC: NCBI Reference Sequence: CAA25015.2

1NKPA identity $=100 \%$

1NLWA identity $=28 \%$

1AM9D identity $=34 \%$

BRCA1: NCBI Reference Sequence: AAH30969

Templates

1JM7A identity $=100 \%$

2JDQA identity $=34 \%$

$1 \mathrm{~V} 87 \mathrm{~A}$ identity $=45 \%$

p53: NCBI Reference Sequence: BAC16799

Templates

2FEJA identity $=99 \%$

3EXJA identity $=87 \%$

2RMNA identity $=49 \%$

EGFR: NCBI Reference Sequence: AAI18666 Templates

$1 \mathrm{~N} 8 \mathrm{YC}$ identity $=44 \%$

2A91A identity $=45 \%$

3I 2 TA identity $=40 \%$

\section{RESULTS}

The Modeller 9v7 generated models (best models analyzed by Ramachandran Plot) are as follows: 


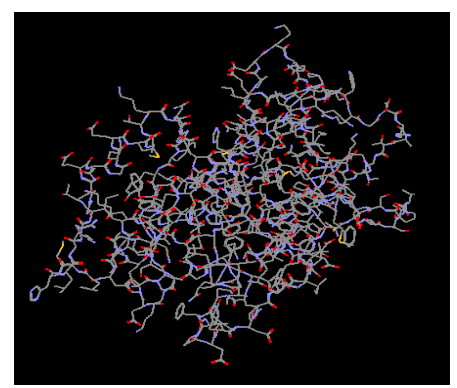

Fig 14. Structure of RAS (visualization in RASMOL)

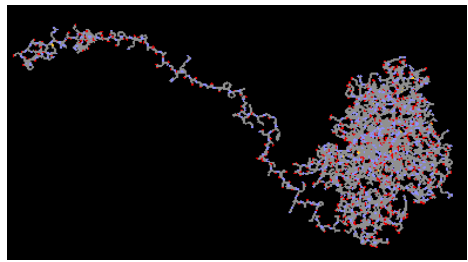

Fig 15. Structure of MYC (visualization in RASMOL)

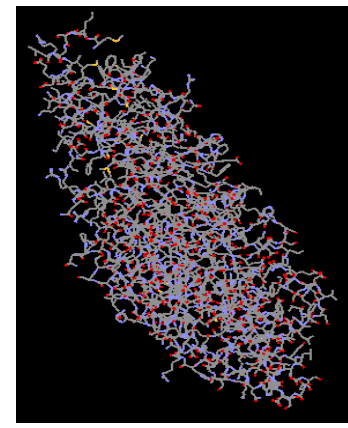

Fig 16. Structure of SRC (visualization in RASMOL)

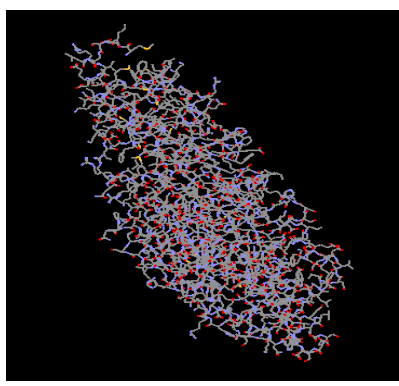

Fig 17. Structure of BRCA1 (visualization in RASMOL)

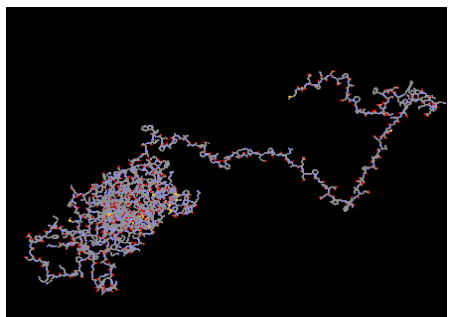

Fig 18. Structure of P53(visualization in RASMOL)

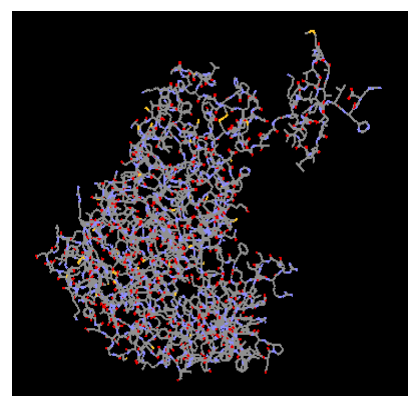

Fig 19. Structure of EGFR (visualization in RASMOL)

The results of Ramachandran plot is as follows:

1) RAS

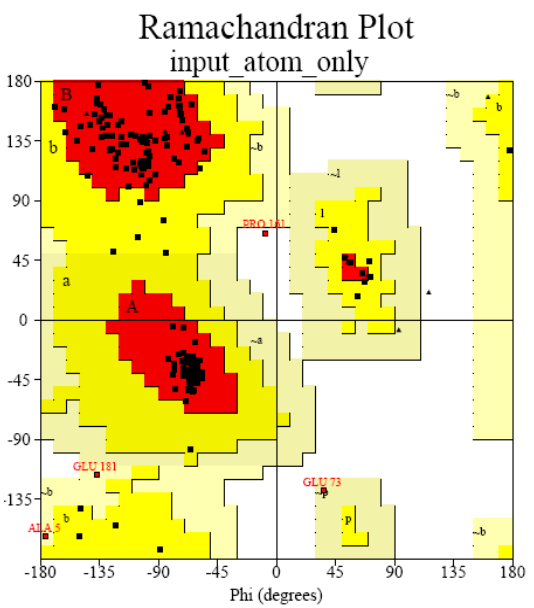

Plot statistics

$\begin{array}{lrc}\text { Residues in most favoured regions }[\mathrm{A}, \mathrm{B}, \mathrm{L}] & 165 & 88.2 \% \\ \text { Residues in additional allowed regions }[\mathrm{a}, \mathrm{b}, \mathrm{l}, \mathrm{p}] & 19 & 10.2 \% \\ \text { Residues in generously allowed regions }[\mathrm{a}, \sim \mathrm{v}, \sim \mathrm{l}, \sim \mathrm{p}] & 3 & 1.6 \% \\ \text { Residues in disallowed regions } & 0 & 0.0 \% \\ & \ldots-. & \ldots \\ \text { Number of non-glycine and non-proline residues } & 187 & 100.0 \% \\ \text { Number of end-residues (excl. Gly and Pro) } & 2 & \\ \text { Number of glycine residues (shown as triangles) } & 10 & \\ \text { Number of proline residues } & 9 & \\ & \ldots . . & \\ \text { Total number of residues } & 208 & \end{array}$

Fig 20. Ramachandran Plot analysis of RAS (best model)

2) BRAC1

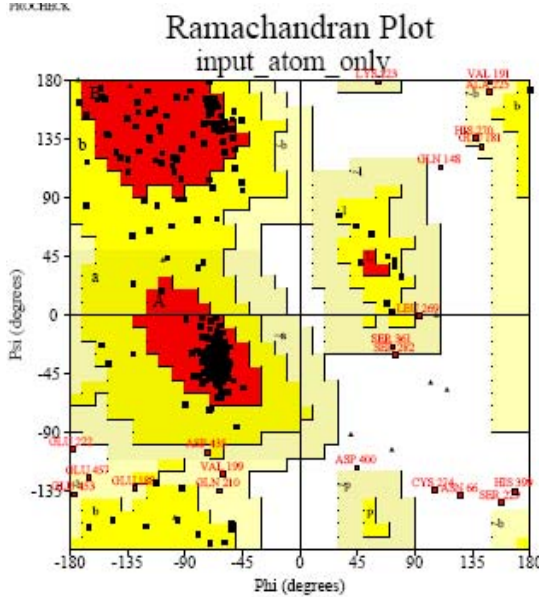


Plot statisics

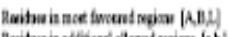

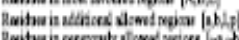

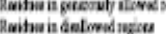

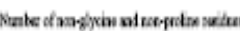

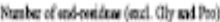

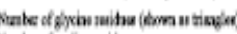

Nente of pulu natau

Tollunte of waitum

Fig 21. Ramachandran Plot analysis of BRCA1 (best model)

3) MYC

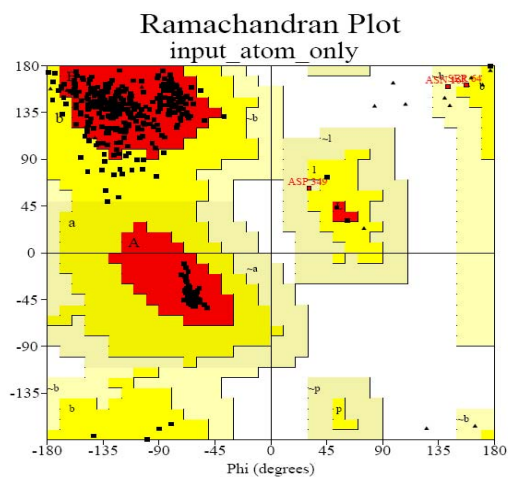

Plot statistics

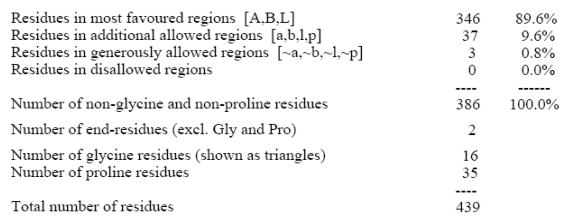

Fig 22. Ramachandran Plot analysis of MYC (best model)

4) $\mathrm{SRC}$

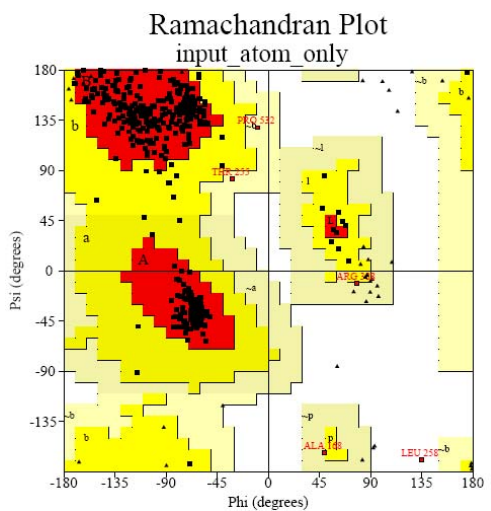

Plot statistics

\begin{tabular}{|c|c|}
\hline $\begin{array}{l}\text { Residues in most favoured regions }[\mathrm{A}, \mathrm{B}, \mathrm{L}] \\
\text { Residues in additional allowed regions }[\mathrm{a}, \mathrm{b}, \mathrm{l}, \mathrm{p}] \\
\text { Residues in generously allowed regions }[\sim \mathrm{a}, \sim \mathrm{b}, \sim, \sim, \sim \mathrm{p}] \\
\text { Residues in disallowed regions }\end{array}$ & $\begin{array}{r}429 \\
26 \\
3 \\
1\end{array}$ \\
\hline Number of non-glycine and non-proline residues & 459 \\
\hline Number of end-residues (excl. Gly and Pro) & 2 \\
\hline $\begin{array}{l}\text { Number of glycine residucs (shown as triangles) } \\
\text { Number of proline residues }\end{array}$ & $\begin{array}{r}43 \\
32 \\
\ldots-2\end{array}$ \\
\hline Total number of residues & 36 \\
\hline
\end{tabular}

Fig 23. Ramachandran Plot analysis of SRC (best model)

Lingands used for each protein are as follows:

RAS: Limonene, Berberine and Curcumin combination.

BRCA1: Podophyllotoxin, D-Limonene and Sulforaphane combination.

P53: Vindoline, Berberine and Curcumin combination

EGFR: Oleanolic Acid, Ursolic Acid and Emodin combination.
5) P53

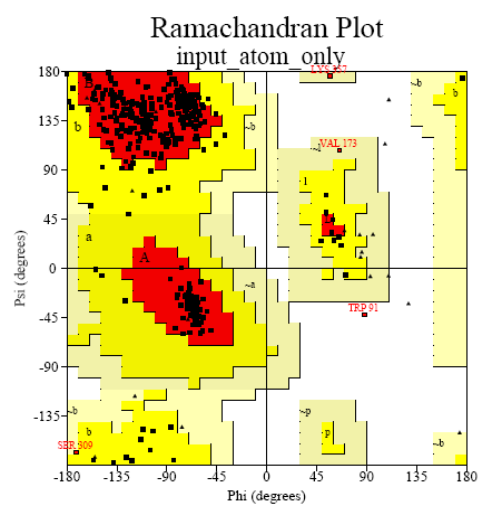

Plot statistics

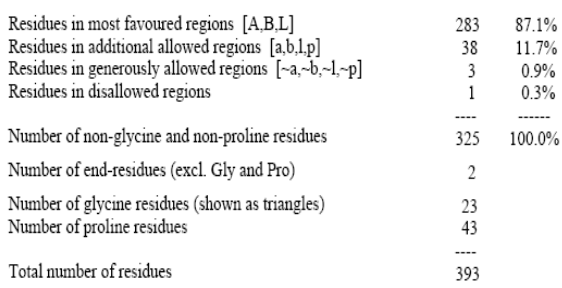

Fig 24. Ramachandran Plot analysis of P53 (best model)

6) EGFR

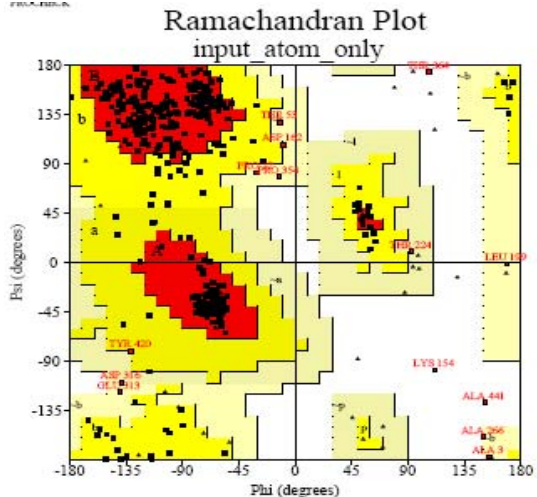

\section{Plot tolitituta}

\begin{tabular}{|c|c|c|}
\hline 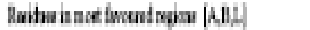 & 313 & 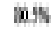 \\
\hline 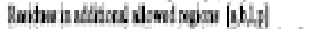 & H & 1645 \\
\hline 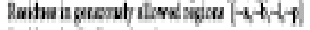 & 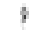 & $2 \mathrm{M}$ \\
\hline \multirow[t]{2}{*}{ Butha in alowdngu } & 1 & $0 \mathbb{W}$ \\
\hline & "me & - \\
\hline 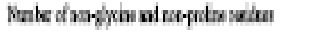 & iii & Itom \\
\hline 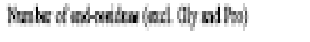 & t & \\
\hline Prake of fhon & 4 & \\
\hline Pakn of plinuatar & II & \\
\hline Tolmentwoin & His & \\
\hline
\end{tabular}

Fig 25. Ramachandran Plot analysis of EGFR (best model)

The proteins were docked with their corresponding ligands by using PATCHDOCK server. The resultant docked structure is as follows:

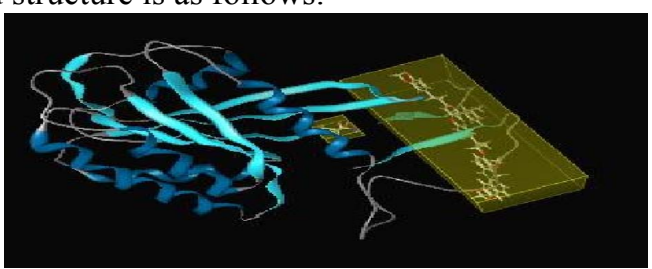

Fig 26. Docked structure of RAS protein with Limonene, Berberine and Curcumin combination as ligand (visualization in ligand scout) 


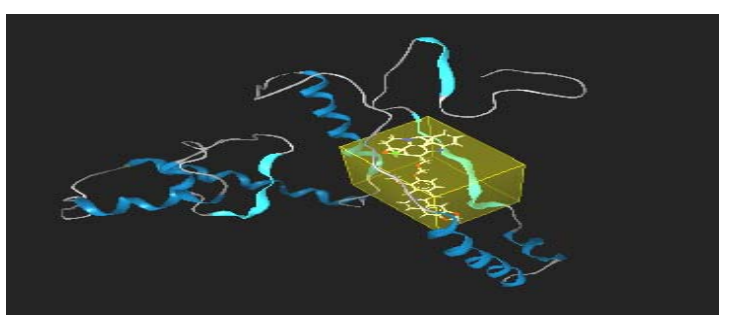

Fig 27. Docked structure of MYC protein with berberine, vindoline combination as ligand (visualization in ligand scout)

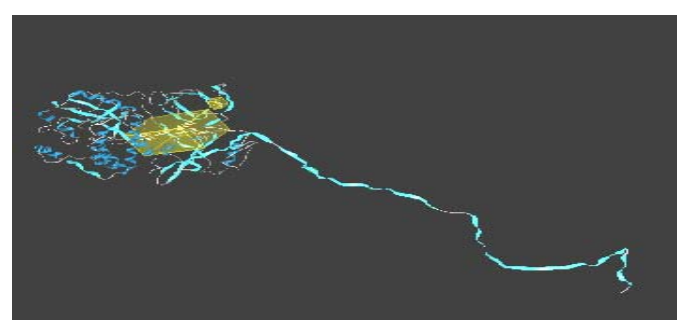

Fig 28. Docked structure of SRC protein with genistein, epcatechin gallate, galangin combination as ligand (visualization in ligand scout)

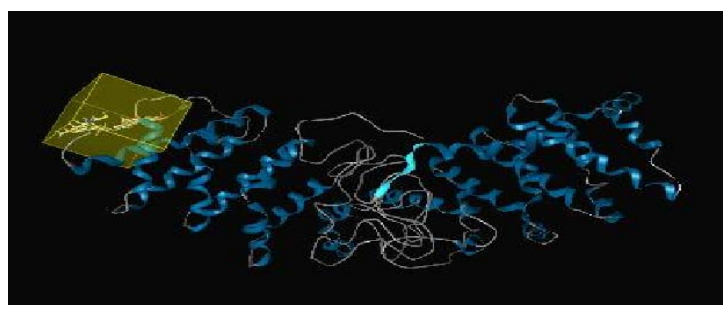

Fig 29. Docked structure of BRCA1 protein with Podophyllotoxin, DLimonene and Sulforaphane combination as ligand (visualization in ligand scout)

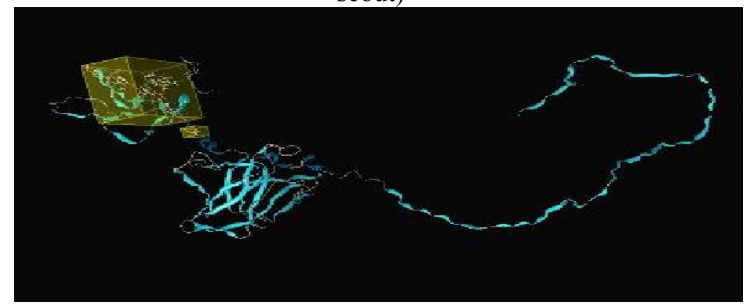

Fig 30. Docked structure of P53 protein with Vindoline, Berberine and Curcumin combination as ligand (visualization in ligand scout)

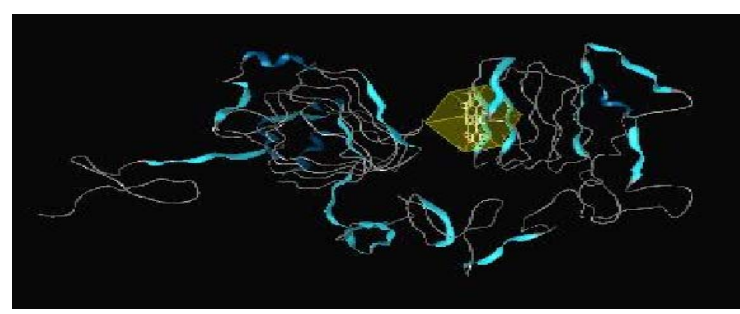

Fig 31. Docked structure of EGFR protein with Oleanolic Acid, Ursolic Acid and Emodin combination as ligand (visualization in ligand scout)

\section{CONCLUSIONS AND Discussions}

The present study was based on the disease "Bronchial carcinoma" which is a cancerous tumor of lungs. It is found out that the ligands (combination of active compounds) docks with the corresponding proteins. The ligand found to bind the protein in an effective manner and this can be effective in treating bronchial carcinoma.

Since the above work is an in-silico work, the compound combinations has to go to clinical trials to establish its efficacy. Again, it is found out the herbs and the plants taken into account have importance in ayurvedic medications to cure tumors of lungs.

\section{REFERENCES}

[1] Bal Govind Tiwari \& B N Upadhyay, "Concept of aging in Ayurveda," Indian Journal of Traditional Knowledge, vol. 8, July 2009, pp. 396-399.

[2] Wang L, Li L,Zhu Y, Fang F, Zuo J, "Proto-oncogene point mutation of c-Ha-ras in bronchogenic carcinoma," Zhongguo Yi Zue Ke Xue Yuan Xue Bao, vol 17, Aug 1995, pp. 281-285.

[3] Yong-song Guan, Yuan Liu,Qing He, Lin Yang, Long Sun, "Combining p53 gene therapy with bronchial artery infusion inhibits growth of non-small cell lung cancer, a case report," Cancer Therapy, vol 5, 2007, pp. 511-514

[4] Melanie Barbara Boettger, Consolato Sergi and Peter Meyer, "BRCA $1 / 2$ mutation screening and $\mathrm{LOH}$ analysis of lung adenocarcinoma tissue in a multiple-cancer patient with a strong family history of breast cancer," J Carcinog, vol. 2, Oct 2003, pp. 1-5

[5] Maximilian Reiter, Christian Welz, Philipp Baumeister, Sabina Schwenk-Zieger and Ulrich Harresu, "Mutagen sensitivity and DNA repair of the EGFR gene in oropharyngeal cancer," Oral Oncology, vol. 46, July 2010, pp. 519-524

[6] M N Gould, "Cancer chemoprevention and therapy by monoterpenes," vol. 105, June 199, pp. 977-979.

[7] Savio Szeto, Christine Miu Ngan Yow, and Ka Wah Fung, "Characterization of Berberine on Human Cancer Cells in Culture," Turk J Med Sci, vol. 32, 2002, pp. 363-368.

[8] Chih-Li Lin and Jen-Kun Lin, "Curcumin: a Potentia Cancer chemopreventive Agent through Suppressing NF- kB Signaling," Journal of Cancer Molecules, vol. 4, 2008, pp. 11-16.

[9] ML de Lemos, "Effects of soy phytoestrogens genistein and diadzein on breast cancer growth," The Annals of Pharmocotherapy, vol. 35, 2001, pp. 1118-1121.

[10] Teruhiro Utsugi, Jiro shibata, Yoshikazu sugimoto, Kumio Aoyagi, Konstanty Wierzba, Takashi Kobunai, Tadafumi Terada, Tomoko Oh-hara, Takashi Tsuruo and Yuji Yamada, "Antitumor Activity of a Novel Podophyllotoxin Derivative (TOP-53) against Lung Cancer and Lung Metastatic Cancer," Cancer Research, vol. 56, June 1996, pp. 2809-2814.

[11] Steven J.T. Jackson and Keith W. Singletary, "Sulforaphane Inhibits Human MCF-7 Mammary Cancer Cell Mitotic Progression and Tubulin Polymerization,” J. Nutr., vol. 134, Sept. 2004, pp. 22292236.

[12] Geng-Shuen Wu, Thomas Nabih, Leisa Youel, Wanda PeczynskaCzoch, and John P. Rosazza, "Microbial Transformations of Natural Antitumor Agents:O-Demethylation of Vindoline by Sepedonium," Antimicrob Agents Chemother., vol. 14, Oct 1978, pp. 601-604.

[13] Qing Huang, Han-Ming Shen, Guanghou Shui, Markus R. Wenk, and Choon-Nam Ong, "Emodin Inhibits Tumor Cell Adhesion through Disruption of the Membrane Lipid Raft-Associated Integrin Signaling Pathway" Cancer Research, vol. 66, June 2006, pp. 5807-5815.

[14] Shishir Shishodia, Sekhar Majumdar, Sanjeev Banerjee and Bharat B Aggarwal, "Ursolic Acid Inhibits Nuclear Factor-kB Actication Induced by Carcinogenic Agents through Suppression of $1 \mathrm{~kb} \alpha$ Kinase and p65 Phosphorylation: Correlation with Down-Regulation of Cyclooxygenase 2, Matrix Metalloproteinase 9, and Cyclin D11," Cancer Research, vol. 63, August 2003, 4375-4383.

[15] Jie Liu, "Oleanolic acid and ursolic acid: Research Perspective," Journal of Ethnopharmacology, vol. 100, August 2005, pp. 92-94.

[16] Coward L, Barnes NC, Setchell KDR, Barnes S (1993). "Genistein, daidzein, and their $\beta$-glycoside conjugates: antitumor isoflavones in soybean foods from American and Asian diets". J Agric Food Chem 41 (11): 1961-1967.

[17] Shiota S, Shimizu M, Mizushima T, Ito H, Hatano T, Yoshida T, Tsuchiya "Marked reduction in the minimum inhibitory concentration (MIC) of beta-lactams in methicillin-resistant Staphylococcus aureus produced by epicatechin gallate, an ingredient of green tea (Camellia sinensis)", T.Biol Pharm Bull. 1999 Dec;22(12):1388-90.

[18] Ciolino, H P; Yeh, G C (1999). "The flavonoid galangin is an inhibitor of CYP1A1 activity and an agonist/antagonist of the aryl hydrocarbon receptor". British Journal of Cancer 79 (9/10): 13401346 